\title{
A Census of Baryons in Galaxy Clusters and Groups
}

\author{
Anthony H. Gonzalez ${ }^{1}$, Dennis Zaritsky ${ }^{2,3}$ \\ and Ann I. Zabludoff ${ }^{2,3}$ \\ ${ }^{1}$ Department of Astronomy, University of Florida, Gainesville, FL 32611-2055, USA \\ email: anthony@astro.ufl.edu \\ ${ }^{2}$ Steward Observatory, University of Arizona, 933 North Cherry Avenue, \\ Tucson, Arizona 85721, USA \\ ${ }^{3}$ Center for Cosmology and Particle Physics, Dept. of Physics, NYU, \\ New York, NY, 10003, USA
}

\begin{abstract}
While the baryon fraction in galaxy groups and clusters may be expected to reflect the universal value, observations of cluster baryon fractions have generally fallen short of this expectation and indicated a possible correlation with cluster mass. We present a new determination of the total baryon budget in nearby galaxy groups and clusters that includes the contributions from stars in galaxies, intracluster stars, and the intracluster medium. We find that the baryon mass fraction within $r_{500}$ is independent of system mass and lower than the WMAP value. We conclude however that the present shortfall provides no compelling evidence for additional missing baryons, since it may arise due to a theoretically predicted deficit of baryons within $r_{500}$ and systematic uncertainties associated with the mass determinations. With the addition of the ICL to the stellar mass in galaxies, the increase in X-ray gas mass fraction with increasing total mass is entirely accounted for by a decrease in the total stellar mass fraction, supporting the argument that the behavior of both the stellar and X-ray gas components is dominated by a decrease in star formation efficiency in more massive environments. Within just the stellar component, the fraction of the total stellar luminosity in the central, giant brightest cluster galaxy (BCG) and ICL (hereafter the BCG+ICL component) decreases as velocity dispersion $(\sigma)$ increases, suggesting that ICL may grow less efficiently in higher mass environments. The identification of low mass groups with large BCG+ICL components also demonstrates that the massive cluster environment is not required to form intracluster stars. These proceedings are a condensed version of the work presented in Gonzalez, Zaritsky \& Zabludoff (2007), and we refer the reader to that paper for a more complete discussion.
\end{abstract}

Keywords. galaxies: clusters: general, galaxies: elliptical and lenticular, cD, galaxies: fundamental parameters, cosmology: observations

\section{Introduction}

For any of the astrophysical systems discussed during this conference, the first step in determining whether there are "lost baryons" is a full accounting of the known baryonic constituents. In galaxy clusters and groups the baryon fraction is expected to reflect the universal value (see White et al. 193; Ettori et al. 2006), but baryon accountings in clusters to date have fallen short of this expectation (Ettori 2003; McCarthy et a. 2006) and uncovered possible intriguing trends with cluster mass (Lin et al. 2003). With the precise measurement of the universal baryon fraction from WMAP (Spergel et al. 2006), this shortfall has gained in physical significance, leading to suggestions that physical processes lower the cluster baryon fraction relative to the Universe (see, for example, He 
et al. 2005), that there may be significant, undetected baryon components (see Ettori 2003; Lin et al. 2004b), or that WMAP underestimates $\Omega_{m}$ (McCarthy et al. 2006).

One baryonic component that past studies were unable to include directly as a function of mass is the intracluster stars (hereafter the intracluster light or ICL). In recent years, multiple studies have detected intracluster light via stacking of large cluster samples (Zibetti et al. 2005), deep observations of individual clusters (Feldmeier et al. 2004; Gonzalez et al. 2005; Krick et al. 2006; Seigar et al. 2007), and even identification of individual intracluster stars in very nearby clusters (Durrell et al. 2002; Feldmeier et al. 2004a; Aguerri et al. 2005; Ciardullo et al. 2005; Gerhard et al. 2005). The general consensus is now that all clusters have an ICL component. The natural extension of these studies is placing the ICL in the larger context of cluster and group evolution, examining directly whether this component is important in the chemical enrichment of the intragalactic medium (Lin et al. 2004b; Zaritsky et al. 2004), the total stellar budget, or even in the total baryon budget. While recent work suggests that the ICL does not contribute much to the overall baryon budget of the most massive clusters (the ones most often observed), it could become important in lower mass systems where the plasma is less dominant and the apparent discrepancy between observations and the WMAP baryon fraction is most severe (e.g. Lin et al. 2003)). This issue is mostly unexplored, as previous ICL measurements have come either from a few systems with little dynamic range (Feldmeier et al. 2004b; Krick et al. 2006) or from stacked systems for which mass estimators have been crude (Zibetti et al. 2005). Here we present a new study in which we include the ICL's contribution to the baryon budget directly, explore the dependence of baryon fraction on cluster mass, and quantify the relative importance of different stellar and baryonic components. $\dagger$

\section{An Intracluster Light Survey}

The current work is part of an ongoing survey of intracluster light in nearby clusters and groups. In Gonzalez et al. (2005) we presented a sample of 24 clusters and groups at $0.03<z<0.13$ for which we obtained drift scan imaging and demonstrated that the brightest cluster galaxy and intracluster light are structurally distinct. These systems were chosen to contain a dominant brightest cluster galaxy (BCG) and span a range of velocity dispersions from poor groups to rich clusters. We have used this sample to assess the importance of intracluster stars in the chemical enrichment of the ICM (Zaritsky et al. 2004) and demonstrate that all galaxy spheroids including the spheroid of intracluster stars lie on a single fundamental manifold (Zaritsky et al. 2006). In the latter paper we also present velocity dispersions for 23 of these clusters and groups. In Gonzalez et al. (2007), we use the above data sets to quantify the properties of the intracluster light as a function of cluster mass and radius, and we conduct a census of the total baryon content in clusters and groups including the contribution of intracluster stars. It is the results from this latter work that we recap in these proceedings.

\section{Luminosity, Stellar Mass, and Mass}

We estimate the total baryon fraction within $r_{500}$, which is the largest radius for which the current X-ray data require no model extrapolation (Vikhlinin et al. 2006). The systems in our ICL sample have measured velocity dispersions, but generally lack X-ray data. To obtain $r_{500}$ and $\mathcal{M}_{500}$ values for the clusters in our ICL sample that are directly

$\dagger$ We assume $\Omega_{M}=0.27, \Omega_{\Lambda}=0.73$, and $H_{0}=70 \mathrm{~km} \mathrm{~s}^{-1}$ throughout. 
comparable to those determined in X-ray studies, we therefore derive calibrations of the $\sigma-r_{500}$ and $\sigma-\mathcal{M}_{500}$ relations using the clusters from Vikhlinin et al. (2006) that also have published velocity dispersions (Girardi et al. 1998; Wu et al. 1999).

\subsection{Total Luminosities}

We determine the luminosity of the BCG+ICL component as follows. We used the GALFIT package (Peng et al. 2002) to fit the surface brightness distribution in each cluster out to a radius of $300 \mathrm{kpc}$ from the BCG. The BCG and ICL were best fit with separate $r^{1 / 4}$ profiles. Here we use those best-fit profiles to construct a 2-D model image from which we determine the flux within circular apertures corresponding to $r_{500}$ and to fractions of $r_{200}$.

We compute the total luminosity of non-BCG cluster galaxies within the same apertures $\left(r_{500}\right.$ and fractions of $\left.r_{200}\right)$ used for the BCG+ICL. Hereafter, we refer to the non-BCG cluster galaxies as simply "cluster galaxies". We sum the flux of all galaxies fainter than the BCG and brighter than $m_{I}=18$ lying within the aperture, perform a statistical background subtraction, and include a completeness correction to account for the contribution of fainter cluster members.

\subsection{Total Stellar Masses}

The conversion from luminosity to stellar mass for the cluster galaxies and the BCG+ICL potentially incurs the most significant uncertainty in the total baryon mass budget. To estimate a mean $M / L$, we use the SAURON results from Cappellari et al. (2006), which provide luminosity dependence of the central stellar mass-to-light ratio. We compute a luminosity-weighted $\mathrm{M} / \mathrm{L}$ for $L>0.25 L_{*}$ (the range over which the SAURON relation is determined) using the Christlein \& Zabludoff (2003) luminosity function. We adopt the resultant value, $M / L=3.6$, for both the cluster galaxies and the BCG+ICL in our baryon calculation, but note that the uncertainty is at least $10 \%$ and that $M / L$ varies with both mass (Cappellari et al. 2006; Zaritsky et al. 2006) and stellar population. An improved accounting for the ICL component would require knowledge of the source of the ICL stars if the variations in $M / L$ are driven by population, rather than dynamical, differences. An improved accounting for the cluster galaxy population would require determining the fraction of spiral galaxies in these clusters and using a more appropriate $M / L$ for the spirals.

\subsection{Total Mass in the Intracluster Medium}

Determination of total cluster baryon fractions also requires measurement of the mass of hot gas in the intracluster medium. Because we lack these data for clusters in our sample, we fit the behavior of the stellar mass fraction with cluster mass and use this relation to derive total baryon fractions for clusters with published X-ray gas fractions. For the gas mass fractions we adopt the values of X-ray gas fraction inside of $r_{500}, f_{g}$, and of enclosed mass, $\mathcal{M}_{500}$, from the tabulations by Vikhlinin et al. (2006) and Gastaldello et al. (2006).

\section{Baryon Mass Fractions}

Summing the three baryonic components - the hot gas of the intracluster medium, the stars of the BCG+ICL, and the stars of the other cluster galaxies - gives a total baryon mass fraction, $f_{b}$, that is constant as a function of total mass (Figure 1). This constancy arises in large part from the inclusion of the ICL for systems with $M_{500}<$ few $\times 10^{14} M_{\odot}$. The weighted mean value of the total baryon mass fraction is $f_{b}=0.133$. 
$\sigma[\mathrm{km} / \mathrm{s}]$

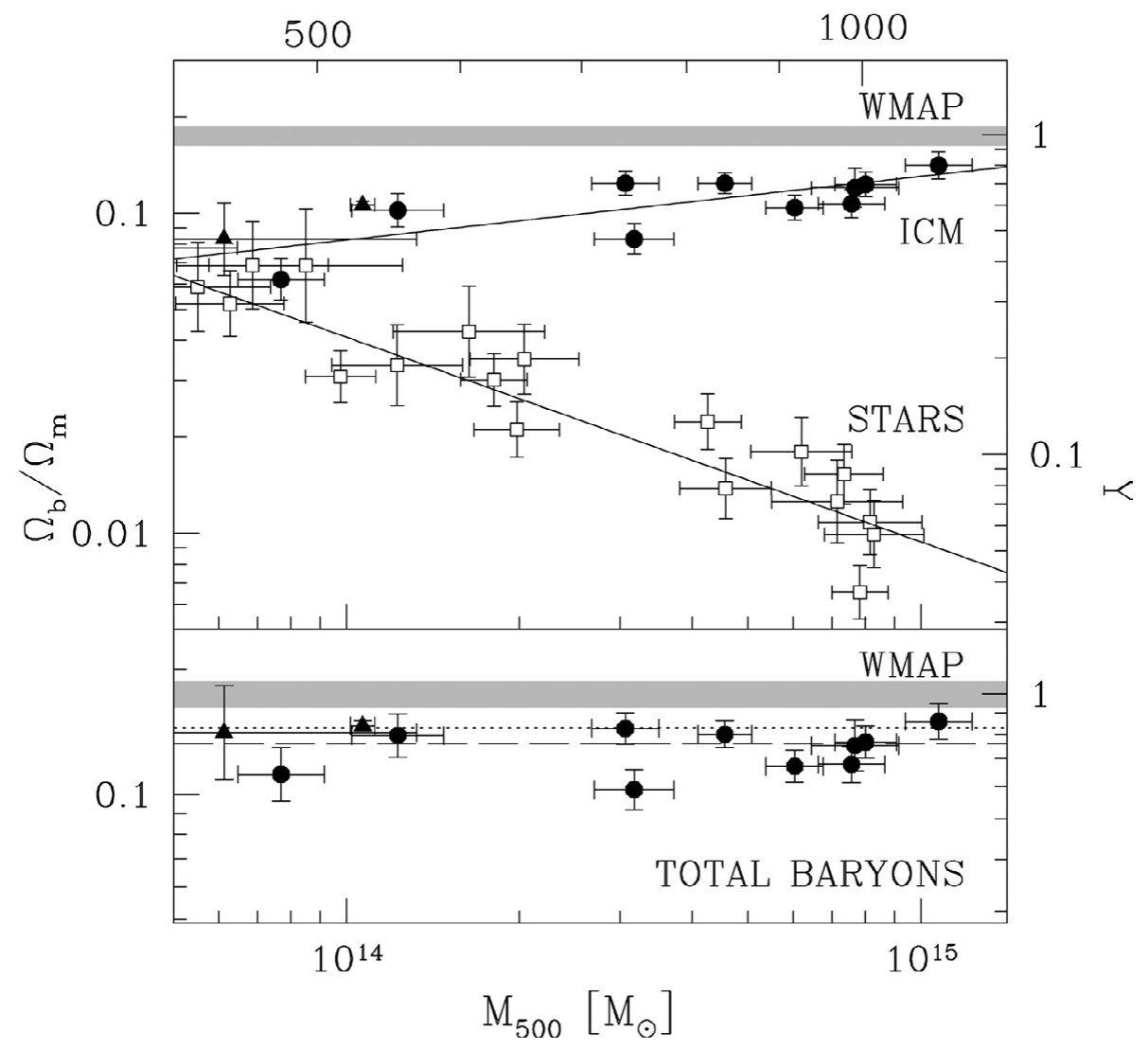

Figure 1. Baryon fractions within $r_{500}$ as a function of $\mathcal{M}_{500}$ (bottom axis) and velocity dispersion (top axis). Upper panel: Data from Vikhlinin et al. (2006) (filled circles) and Gastaldello et al. (2006) (filled triangles) for the X-ray gas mass fraction within $r_{500}$, and the stellar mass fraction (BCG+ICL+galaxies, open squares), as described in the text, for systems in our sample with masses that overlap the range shown for the X-ray studies. Overplotted are the best fit relations for the Vikhlinin et al. (2006) sample gas mass fractions and for our stellar mass fractions. The WMAP $1 \sigma$ confidence region for the universal baryon fraction from Spergel et al. (2006) is shown for comparison, and the right-hand axis shows $Y$, the ratio of the baryon fraction for each component to the universal value from WMAP. Lower panel: The total baryon fraction derived for the Vikhlinin et al. (2006) and Gastaldello et al. (2006) clusters if our best-fit stellar baryon relation is used to estimate a stellar baryon contribution for each of these systems. The dashed line corresponds to the weighted mean for the sample, which is $\Omega_{b} / \Omega_{m}=0.133 \pm 0.004$. We observe no trend in baryon fraction with cluster mass. The error bars on the individual data points include only statistical uncertainties. The dotted line, which shows the unweighted mean for the combined Zhang et al. (2007) and Rasmussen \& Ponman (2006) samples, is included to provide a sense of the systematic uncertainties.

The plasma component, which dominates at cluster masses $>10^{14} M_{\odot}$, has a shallower dependence on mass than the stellar component. We use our best fit relation for the stellar mass to convert the X-ray gas fractions from Vikhlinin et al. (2006) and Gastaldello et al. (2006) to total baryon fractions on a cluster-by-cluster basis. The resulting total baryon fractions, shown in the lower panel of Figure 1, are independent of cluster mass over the range of $\mathcal{M}_{500}$ from $6 \times 10^{13}$ to $10^{15} \mathrm{M}_{\odot}$. If one considers only the statistical uncertainties 
associated with our total cluster baryon fraction and the WMAP measurement, then the two values are discrepant at the level of $3.2 \sigma$.

There are four possible interpretations of this result in the context of a full baryon accounting. First, systematic uncertainties may be significantly larger than the random errors, and our measurement may therefore be consistent with the universal WMAP value. Because the gas mass fraction is much larger than the stellar mass fraction over the majority of the mass range we probe, we focus this discussion on potential errors in the gas mass fraction. Systematic errors, by their nature, are often difficult to calculate and can best be illuminated by comparing independent studies. Zhang et al. (2007) and Rasmussen \& Ponman (2006) provide gas mass fractions for high and low mass systems, respectively. Zhang et al. (2007) find gas fractions that are $15 \%$ larger than those we adopted over the range probed by their sample. Rasmussen \& Ponman (2006) also find gas fractions that are larger than those we adopted for comparably low mass systems. Together, these two samples cover the mass range of our adopted sample and result in an average $f_{b}=0.149$, which is $2.1 \sigma$ discrepant with the WMAP value and $12 \%$ larger than the $f_{b}$ we derive for the Vikhlinin et al. (2006) data set. We conclude that indeed the systematic uncertainties dominate, that the sense of the uncertainty works to diminish the discrepancy between measurements and expectations, and that the case for undetected baryon components rests on removing these systematic uncertainties. Second, the baryon mass fraction with $r_{500}$ in clusters may be different from the universal value. Cluster simulations routinely predict baryon fractions that are lower than the universal baryon fraction by $\sim 10 \%$. We have not adopted any of those as the target because of uncertainty in the precise correction, but this factor acts to reduce the dsicrepancy. Third, it is possible as argued by McCarthy et al. (2006) that the actual universal baryon fraction may be lower than the WMAP value. Fourth, the shortfall may be resolved by yet undetected baryon components. The constancy of the measured baryon fraction with total system mass argues against a large undetected component, but one could contribute at a modest level and bypass current detection. In the end, all of these possibilities may contribute at some level to a final resolution of the baryon accounting, but at this point we are left to conclude that there is currently no compelling evidence for undetected baryons.

\section{Properties of the Intracluster Light}

In Figure 2 we determine the fraction of total stellar luminosity contained in the $\mathrm{BCG}+\mathrm{ICL}$ component as a function of velocity dispersion. The existence of groups in which the fraction of stars in the BCG+ICL exceeds that in galaxies out to $r_{500}$ (Figure 2) demonstrates that the rich cluster environment is not a requirement for the production of a significant intracluster stellar population. Instead, it appears that local density maxima in both the group and cluster environments are capable of generating intracluster stars, presumably via tidal processes. This conclusion is valid whether one is interested in the combination of the BCG and ICL, or in the ICL alone, because the latter contains the bulk (typically $>80 \%$ ) of the total light in the two components (Gonzalez et al. 2005). However, our results should not be interpreted to mean that all groups have a significant ICL component, because our original sample selection focused on systems with dominant BCGs.

We also explore the radial dependence of the intracluster light. For the full ensemble of clusters the BCG+ICL luminosity fraction smoothly declines from $65 \%$ within $0.1 r_{200}$ to $33 \%$ within $r_{200}$. This radial, which demonstrates that the galaxies are less concentrated 


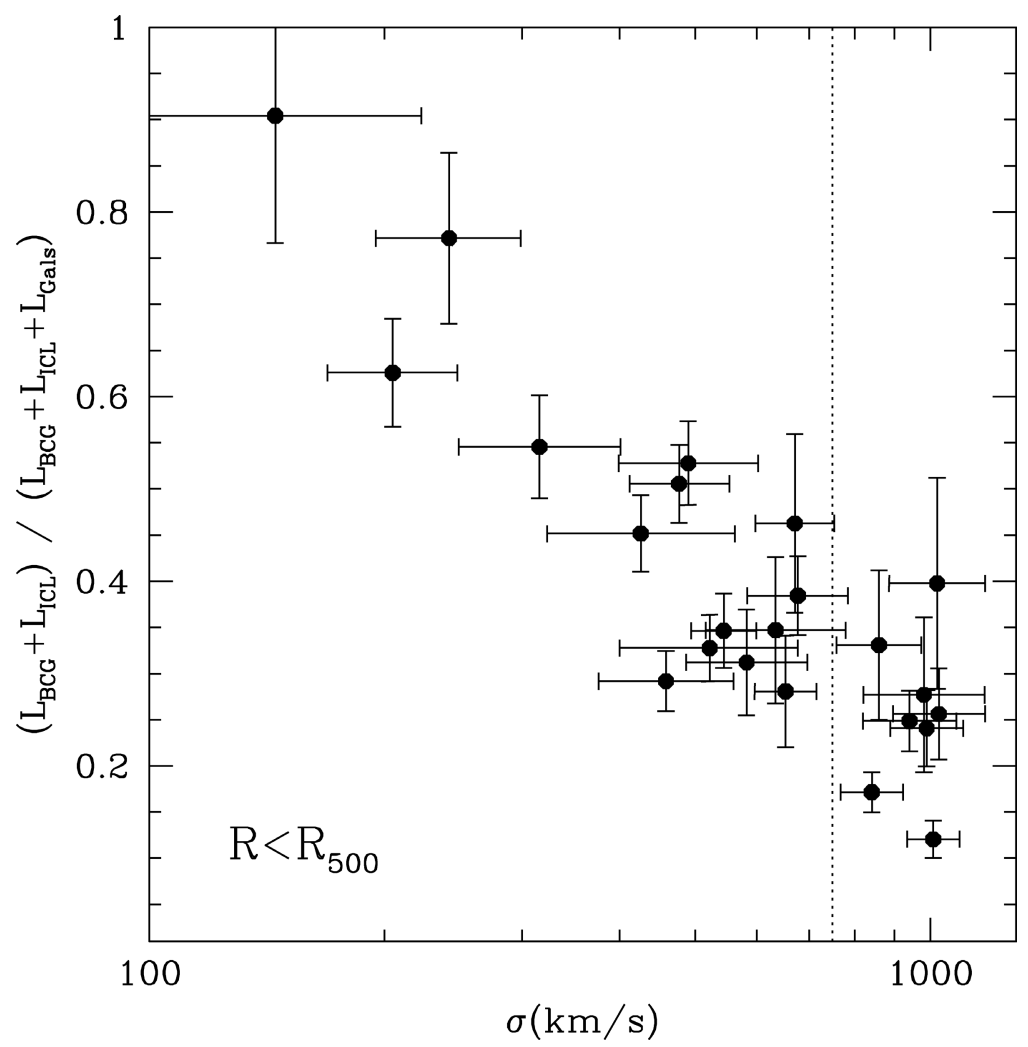

Figure 2. The fraction of the total stellar luminosity, measured within $r_{500}$, which is contained in the $\mathrm{BCG}+\mathrm{ICL}$ component as a function of cluster velocity dispersion. The dotted vertical line corresponds to $\sigma=750 \mathrm{~km} \mathrm{~s}^{-1}$, roughly the median velocity dispersion for an Abell richness class $R \geqslant 1$ cluster (Zabludoff et al. 1990).

than the ICL, is also seen by Zibetti et al. (2005 and these proceedings) and is predicted by the simulations of Murante et al. (2004).

\section{Origin of the Baryon Trends}

We have identified two independent trends with mass that shed light on the environmental dependencies of physical processes that distribute baryons among the various phases. First, we find that the fraction of stars in the ICL grows (and that in galaxies diminishes) with decreasing system mass. This result is predicated on the assumption that we are not strongly biased in the type of system observed, as discussed above. Even so, we now know that in at least some low mass systems the ICL forms efficiently. As a result, we can rule out cluster-only processes, such as harassment (Moore et al. 1996,1998), as the dominant ICL formation mechanism, and find support for mechanisms that would be more effective in lower mass systems, such as galaxy-galaxy interactions Merritt 1984 . Such tidal interactions, which could strip stars from galaxies and build up the ICL, are favored in lower mass groups, because the internal velocity dispersions of group galaxies are similar to the velocity dispersion of the group as a whole. If all low mass systems do have high ICL luminosity fractions, and some are the building-blocks of clusters, then a diluting mechanism, such as the infall of individual galaxies into clusters, would be necessary to reduce the final ICL fraction of more massive systems. Of course, it may 
be that not all low mass groups have high ICL fractions like those in our sample. The low ICL fractions of high mass clusters may therefore simply reflect their growth by the accretion of groups with lower ICL fractions than we observe. A related possibility is that the clusters accrete and mix progenitor groups before they have time to form substantial ICL via galaxy-galaxy interactions, whereas the ICL continues to grow in more isolated groups. Simulations of the formation of the ICL must reproduce these trends and will lead to a better understanding of the details of the formation mechanisms (Willman et al. 2004; Murante et al. 2004; Sommer-Larsen et al. 2005; Sommer-Larsen 2006; Rudick et al. 2006).

Second, we find that the baryon mass fraction across the range of systems for which we have both optical and X-ray data is roughly constant over the mass range $6 \times 10^{13} \mathrm{M}_{\odot}<$ $\mathcal{M}_{500}<1 \times 10^{15} \mathrm{M}_{\odot}$. This result requires a fairly well-tuned balance between the $\mathrm{X}$ ray gas mass fraction, which declines by a factor of two between systems with $\mathcal{M}_{500}$ of $10^{15} M_{\odot}$ and $10^{14} M_{\odot}$, and the stellar mass fraction, which increases by a factor of four to compensate. The matching of the two trends supports the suggestion (Bryan 2000; Lin et al. 2003) that the X-ray gas mass fraction behavior is due to the decreased efficiency of turning gas into stars in the more massive environments. The reduction in star formation efficicency in higher mass systems may arise from the decreased efficiency of tidal interactions among galaxies, the removal of the gaseous reservoirs of galaxies by interactions with the intracluster medium, or increased feedback processess that quench star formation. As usual, interpreting such trends is complicated by the fact that today's lower mass systems are not necessarily similar to the lower mass antecedents of today's higher mass systems.

\section{Conclusions}

We determine the luminosities of the different stellar constituents (including stars associated with cluster galaxies and the intracluster stars) as a function of cluster velocity dispersion $(\sigma)$, cluster mass $\left(\mathcal{M}_{500}\right)$, and cluster-centric radius. Combining these results with current measurements of the X-ray gas mass as a function of $\sigma$, we calculate the dependence of the baryon budget on environment. The main conclusions are:

1. The total baryon mass fraction within $r_{500}$ is nearly constant for systems with $\mathcal{M}_{500}$ between $6 \times 10^{13}$ and $10^{15} \mathrm{M}_{\odot}$. Although the mean baryon mass fraction is formally $>3 \sigma$ lower than the WMAP measurement, both the observational systematic uncertainties which dominate the formal random errors - and the predicted deficit of baryons in clusters relative to the universal value are on the order of the observed baryon shortfall. We therefore conclude that at present the evidence for undetected baryons is not compelling.

2. The inclusion of the combined light from the brightest cluster galaxy and the intracluster stars (BCG+ICL) becomes increasingly important in the baryon budget as the system mass decreases. For systems with $\mathcal{M}_{500}=10^{14} M_{\odot}$, the BCG+ICL component is nearly $35 \%$ of the total stellar luminosity within $r_{500}$, and the mass of the stellar component including the BCG+ICL and the cluster galaxies is comparable to that of the X-ray gas component. Consequently, the increase in X-ray gas mass fraction with increasing $\mathcal{M}_{500}$ is directly reflected by a decrease in the total stellar mass fraction. The matching of the falling stellar mass and rising gas mass with total mass supports the suggestion made previously (Bryan 2000; Lin et al. 2003) that the star formation efficiency decreases with increasing system mass.

3. Considering only the stellar components, we observe that the fraction of the total stellar luminosity in the BCG+ICL decreases with system velocity dispersion. The origin of this trend is that the luminosity of the BCG+ICL component changes more slowly with 
cluster mass than does the luminosity of cluster galaxies, increasing by only a factor of two between $400 \mathrm{~km} \mathrm{~s}^{-1}$ and $1000 \mathrm{~km} \mathrm{~s}^{-1}$. The presence of large BCG+ICL luminosity fractions in the lower mass systems demonstrates that the rich cluster environment is not required for production of "intracluster" stars.

\section{Comments and Future Work}

While our work demonstrates that any "lost baryons" in clusters must contribution a minor fraction of the total baryon component, we do not claim to find evidence that such a component cannot exist. Rather, we have reached the stage where the statistical uncertainties are dominated by statistical considerations, and the best means of moving beyond the analysis that we have presented is via refined constraints from simulations on the expected baryon fraction within $r_{500}$ coupled with careful analysis of the uncertainties associated with the X-ray total masses. Multiple groups are making progress on both fronts and it will be interesting to see whether subsequent results yield consistency between the observations and theoretical expectations.

In the interim, the next logical step in this analysis is to obtain intracluster light and X-ray data for a single sample of clusters so that the relative baryon content of the stellar and hot gas components can be studied on a case-by-case rather than statistical basis. We have thus far obtained XMM data for a few of the systems in the above sample, and aim to expand this work in the near future. Suresh Sivanandam (Arizona) is also leading an effort to quantify the degree to which the intracluster stars that we detect can enrich the intracluster medium for a subset of systems with both ICL and X-ray data.

\section{Acknowledgements}

We thank Jon Davies and the members of the scientific and local organizing committees for their efforts organizing this meeting.

\section{References}

Aguerri, J. A. L., Gerhard, O. E., Arnaboldi, M., Napolitano, N. R., Castro-Rodriguez, N., \& Freeman, K.-C. 2005, $A J, 129,2585$

Bryan, G. L. 2000, ApJ (Letters), 544, L1

Cappellari, M. et al. 2006, MNRAS, 366, 1126

Christlein, D. \& Zabludoff, A. I. 2003, ApJ, 591, 764

Ciardullo, R., Williams, B. F., Durrell, P. R., Vinciguerra, M., Feldmeier, J. J., Jacoby, G. H., Sigurdsson, S., von Hippel, T., Ferguson, H., Tanvir, N., Arnaboldi, M., Gerhard, O., Aguerri, A., \& Freeman, K. C. 2005, BAAS, 37, 1297

Durrell, P. R., Ciardullo, R., Feldmeier, J. J., Jacoby, G. H., \& Sigurdsson, S. 2002, ApJ, 570, 119

Ettori, S. 2003, MNRAS, 344, L13

Ettori, S., Dolag, K., Borgani, S., \& Murante, G. 2006, MNRAS, 365, 1021

Feldmeier, J. J., Ciardullo, R., Jacoby, G. H., \& Durrell, P. R. 2004a, ApJ, 615, 196

Feldmeier, J. J., Mihos, J. C., Morrison, H. L., Harding, P., Kaib, N., \& Dubinski, J. 2004b, ApJ, 609, 617

Gastaldello, F., Buote, D. A., Humphrey, P. J., Zappacosta, L., Bullock, J. S., Brighenti, F., \& Mathews, W. G. 2006, ArXiv Astrophysics e-prints

Gerhard, O., Arnaboldi, M., Freeman, K. C., Kashikawa, N., Okamura, S., \& Yasuda, N. 2005, ApJ (Letters), 621, L93

Girardi, M., Giuricin, G., Mardirossian, F., Mezzetti, M., \& Boschin, W. 1998, ApJ, 505, 74

Gonzalez, A. H., Zaritsky, D., \& Zabludoff, A. I. 2007, ApJ, 666, in press

Gonzalez, A. H., Zabludoff, A. I., \& Zaritsky, D. 2005, ApJ, 618, 195 
Gonzalez, A. H., Zabludoff, A. I., Zaritsky, D., \& Dalcanton, J. J. 2000, ApJ, 536, 561

He, P., Feng, L.-L., \& Fang, L.-Z. 2005, ApJ, 623, 601

Krick, J. E., Bernstein, R. A., \& Pimbblet, K. A. 2006, AJ, 131, 168

Lin, Y.-T. \& Mohr, J. J. 2004, ApJ, 617, 879

Lin, Y.-T., Mohr, J. J., \& Stanford, S. A. 2003, ApJ, 591, 749

-. 2004, ApJ, 610, 745

McCarthy, I. G., Bower, R. G., \& Balogh, M. L. 2007, MNRAS, 377, 1457

Merritt, D. 1984, ApJ, 276, 26

Moore, B., Katz, N., Lake, G., Dressler, A., \& Oemler, Jr., A. 1996, Nature, 379, 613

Moore, B., Lake, G., \& Katz, N. 1998, ApJ, 495, 139

Murante, G., Arnaboldi, M., Gerhard, O., Borgani, S., Cheng, L. M., Diaferio, A., Dolag, K., Moscardini, L., Tormen, G., Tornatore, L., \& Tozzi, P. 2004, ApJ (Letters), 607, L83

Peng, C. Y., Ho, L. C., Impey, C. D., \& Rix, H.-W. 2002, AJ, 124, 266

Rasmussen, J. \& Ponman, T. J. 2006, ArXiv Astrophysics e-prints

Rudick, C. S., Mihos, J. C., \& McBride, C. 2006, ApJ, 648, 936

Seigar, M. S., Graham, A. W., \& Jerjen, H. 2007, MNRAS, 378, 1575

Sommer-Larsen, J. 2006, MNRAS, 369, 958

Sommer-Larsen, J., Romeo, A. D., \& Portinari, L. 2005, MNRAS, 357, 478

Spergel, D. N. et al. 2006, ApJ

Vikhlinin, A., Kravtsov, A., Forman, W., Jones, C., Markevitch, M., Murray, S. S., \& Van Speybroeck, L. 2006, ApJ, 640, 691

White, S. D. M., Navarro, J. F., Evrard, A. E., \& Frenk, C. S. 1993, Nature, 366, 429

Willman, B., Governato, F., Wadsley, J., \& Quinn, T. 2004, MNRAS, 355, 159

Wu, X.-P., Xue, Y.-J., \& Fang, L.-Z. 1999, ApJ, 524, 22

Zabludoff, A. I., Huchra, J. P., \& Geller, M. J. 1990, ApJS, 74, 1

Zaritsky, D., Gonzalez, A. H., \& Zabludoff, A. I. 2004, ApJ (Letters), 613, L93

-. 2006, ApJ, 638, 725

Zaritsky, D., Schectman, S. A., \& Bredthauer, G. 1996, PASP, 108, 104

Zhang, Y. ., Finoguenov, A., Boehringer, H., Kneib, J. ., Smith, G. P., Czoske, O., \& Soucail, G. $2007, A \& A, 467,437$

Zibetti, S., White, S. D. M., Schneider, D. P., \& Brinkmann, J. 2005, MNRAS, 358, 949 